\title{
sciendo
}

\section{MECHANISM AND FUNCTIONS OF IDENTIFIED miRNAS IN POULTRY SKELETAL MUSCLE DEVELOPMENT - A REVIEW}

\author{
Asiamah Amponsah Collins", Kun Zou, Zhang Li, Su Ying* \\ College of Agriculture, Guangdong Ocean University, Zhanjiang, 524025, PR China \\ \#contribueted equally \\ •Corresponding author: dwkxsy@163.com
}

\begin{abstract}
Development of the skeletal muscle goes through several complex processes regulated by numerous genetic factors. Although much efforts have been made to understand the mechanisms involved in increased muscle yield, little work is done about the miRNAs and candidate genes that are involved in the skeletal muscle development in poultry. Comprehensive research of candidate genes and single nucleotide related to poultry muscle growth is yet to be experimentally unraveled. However, over a few periods, studies in miRNA have disclosed that they actively participate in muscle formation, differentiation, and determination in poultry. Specifically, miR-1, miR-133, and miR-206 influence tissue development, and they are highly expressed in the skeletal muscles. Candidate genes such as CEBPB, MUSTN1, MSTN, IGF1, FOXO3, $m$ TOR, and NFKB1, have also been identified to express in the poultry skeletal muscles development. However, further researches, analysis, and comprehensive studies should be made on the various miRNAs and gene regulatory factors that influence the skeletal muscle development in poultry. The objective of this review is to summarize recent knowledge in miRNAs and their mode of action as well as transcription and candidate genes identified to regulate poultry skeletal muscle development.
\end{abstract}

Key words: poultry, skeletal muscle development, miRNAs, genes, proliferation, differentiation

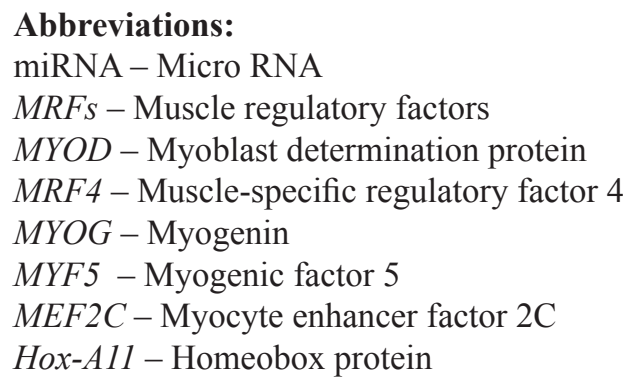


ROCK1 - Rho-associated coiled-coil containing protein kinase 1
MUSTN1 - Musculoskeletal embryonic nuclear protein 1
MSTN - Myostatin
IGF - Insulin-like growth factor
FOXO3 - Forkhead Box 03
mTOR - Mammalian target of rapamycin
HDAC4 - Histone deacetylase
SRF - Serum response factor
PI3K - Phosphoinositide-3-kinase
ANXA2 - Annexin A2
TPM3 - Tropomyosin 3
EIF $2 A K 3$ - Eukaryotic translation initiation factor 2 alpha kinase 3

Skeletal muscle development is a complex and tightly developmental process comprising differentiation of myoblast from the mesodermal precursor cells fused to form mature myotubes. The myotubes also differentiate to form myofibres during embryogenesis (Buckingham, 2006; Luo et al., 2013; Velleman et al., 2010; White et al., 2010). Genetic factors such as transcription factors, gene polymorphism, DNA methylation, and non-coding RNAs work together to control the development of muscle fiber formation from the mesodermal precursor cells (Berkes and Tapscott, 2005; Clop et al., 2006; Luo et al., 2013; Saccone and Puri, 2010). Myoblast formation, determination, and differentiation are regulated by four main muscle-specific regulatory factors $(M R F S)$ which are myoblast determination protein $(M Y O D)$, muscle-specific regulatory factor $4(M R F 4)$, myogenin $(M Y O G)$, and myogenic factor 5 (MYF5) (Braun and Gautel, 2011; Wood et al., 2013). Myocyte enhancer factor 2C (MEF2C), sine oculis homeobox homolog (SIX1-SIX6), and eyes absent (EYA) are other genes that also control skeletal myogenesis (Grifone et al., 2007; Potthoff and Olson, 2007; Wang et al., 2016). The members of MRFs are essential in embryonic muscle development because they control genes expressly involved in muscle growth, differentiation, morphogenesis, and contractility (Braun and Gautel, 2011; Wood et al., 2013).

Poultry is an essential agricultural organism that serves as a primary source of protein worldwide and bridges the evolutionary gap between mammals and other vertebrates (Hillier et al., 2004).

Owing to the high demand of poultry, several genes associated with skeletal muscle development have been identified to enhance its production (Chen et al., 2015; Xu et al., 2017, 2015, 2013 a, b; Zhu et al., 2017).

Recently, extensive studies have shown that miRNAs play vital roles in skeletal muscle development (van Rooij et al., 2008; Luo et al., 2013). Several discovered miRNAs including miR-1, miR-133, and miR-206 are broadly studied to express in muscle tissue. MiRNA-1 and miRNA-133, which are from the same miRNA cistrons are involved in the development and differentiation of skeletal and cardiac muscle (Chen et al., 2006; Gu et al., 2014; Wu et al., 2019) whereas miR-206 precisely expresses in the skeletal muscle (Hak et al., 2006). Also, miR-181 down-regulates the homeobox protein Hox-A11 which inhibits myogenesis to promote myogenesis 
whereas miR-486 is revealed to induce myoblast differentiation by inhibiting Pax 7 while miR-206 regulates embryonic myogenesis by targeting the deleterious controllers of $M Y O D, M Y F S$, and $M Y O G$ in muscle development stages and miR-27b ensures myogenic differentiation by modulating $\operatorname{Pax} 3$. More so, miR-148a downregulates an inhibitor of myogenesis known as Rho-associated coiled-coil containing protein kinase 1 (ROCK1) and regulates muscle differentiation (Crist et al., 2009; Dey et al., 2012; Kablar and Rudnicki, 2000; Naguibneva et al., 2006). Therefore, the aim of this review was to explore the miRNAs and their mode of action, related transcription and candidate genes identified to regulate poultry skeletal muscle development.

\section{Transcription factors and candidate genes identified in poultry skeletal mus- cle development}

The muscle development process is regulated by several genes which coordinate and complement with each other in every stage. Amongst them are the two most important transcription factors: muscle regulatory factors $(M R F)$ and myocyte enhancer factor 2 (MEF2) families which help in the formation and differentiation of muscles (Berkes and Tapscott, 2005; Braun and Gautel, 2011). These two families jointly regulate and essentially express in embryonic muscle development and control the transcription of various myogenic-specific genes involved in muscle development (Braun and Gautel, 2011; Wood et al., 2013). The $M R F$ family which is basic helix-loop-helix $(b H L H)$ proteins includes MYOD, MYOG, MRF4, and $M Y F 5$ while the $M E F 2$ family which is MADS-box transcription factors consists of $M E F 2 A, M E F 2 B, M E F 2 C$ and MEF2D (Potthoff and Olson, 2007; Wood et al., 2013). Except for $M Y F 5$, the others play the central role in the muscle differentiation, that is, myogenin and MRF4 promote myogenic differentiation during myogenesis (Sumariwalla and Klein, 2001; Wood et al., 2013); MYOD plays the determining role for myoblast formation (Ishibashi et al., 2005) whilst differentiation is enhanced through $M E F 2$ interactions with members of the $M R F$ family and other myogenic factors (Potthoff and Olson, 2007).

Apart from the two families mentioned above, several studies have been made to discover genes and molecular mechanisms involved in the increase in skeletal muscle development in poultry which include MUSTN1, MSTN, IGF1, FOXO3, mTOR, NFKB1, and CEBPB (Chen et al., 2015; Xu et al., 2017, 2015, 2013 a, b; Zhu et al., 2017). Musculoskeletal embryonic nuclear protein 1 (MUSTN1) gene, identified in rats in 2002, was mainly found in skeletal muscles and tendons (Hadjiargyrou et al., 2002; Liu et al., 2010). Myostatin (MSTN), otherwise known as growth differentiation factor $8(G D F-8)$, significantly expresses in the development and growth of cardiac muscle and skeletal muscle (Hennebry et al., 2008; Mendias et al., 2008). In chicken, it is revealed that the polymorphisms of MSTN gene have an impact on several traits and their changes are related to regulating abdominal fat weight, birth weight and breast muscle weight ( $\mathrm{Gu}, 2004)$. It is also reported that the variations of MSTN affect breast meat percentage and multiple traits in duck (Junqing et al., 2011; $\mathrm{Xu}$ et al., 2013). The insulin-like growth factor $(I G F)$ system categorized as an indispensable regulatory system controls cell proliferation and differentiation in many poultry and mammalian tissues such as muscle, bone, liver, reproductive organs, and 
the central nervous system (Castigliego et al., 2010; Dupont and Holzenberger, 2003; Harris and Westwood, 2012; Richards et al., 2005; Song et al., 2012; Xu et al., 2012). Forkhead box 03 (FOXO3) is an essential transcriptional regulator that regulates cell proliferation, differentiation, metabolism, apoptosis, and transformation (Accili and Arden, 2004; van der Horst and Burgering, 2007). Overexpression of FOXO3 downregulated the expression of MRFs (MYOD, MYOG, MRF4, and MYF5) which also enhances skeletal muscle proliferation and differentiation suggesting that $F O X O 3$ may play a vital role in myoblast proliferation and differentiation in poultry (Gan et al., 2016; Yin et al., 2014). Mammalian target of rapamycin (mTOR) through Akt/mTOR pathway and nuclear factor kappa-B, subunit 1 (NFKB1) are essential for regulating muscle fiber growth and skeletal mass development, thus inhibiting atrophy in muscles (Bodine et al., 2001; Hunter and Kandarian, 2004). CEBPB regulates multiple genes in response to growth hormones and activates adipogenesis to inhibit myogenesis (Cui et al., 2011; Hache et al., 2007). Several other candidate genes regulate skeletal muscle development; more research should be made to confirm those that function significantly in the development of poultry skeletal muscle.

\section{MicroRNAs identified in poultry skeletal muscle development}

Skeletal muscle development involves several complex processes from the embryonic stage through to the postnatal muscle fiber hypertrophy. During the early stage, mesodermal precursor cells resulting from somites produce the myoblast which fuses to form mature myotubes. The proliferation of myotubes causes it to differentiate into myofibres (Buckingham, 2006; Luo et al., 2013; Velleman et al., 2010; White et al., 2010). After birth, muscle fibers resulting from satellite cell fusion resize to promote muscle growth (Moss and Leblond, 1971). Different studies have been made to identify and characterize several miRNAs related to skeletal muscle development (Andreote et al., 2014; Li et al., 2018), embryo development (Glazov et al., 2008; Hicks et al., 2008), lipogenesis and cell proliferation (Hicks et al., 2010), lung in response to Mycoplasma gallisepticum HS (Zhao et al., 2017) among others in chicken.

Several miRNAs have been established to regulate skeletal muscle development at each step of the developmental process. Some of them individually expressed in skeletal muscle cells whiles others showed during differentiation progress implying that miRNAs specifically target tissue gene expression pattern (Luo et al., 2013). With regards to poultry growth and development, much attention is drawn to different miRNAs found at different periods in the embryo and muscle development. Comparatively, for embryo development and tissue-specific maintenance, most miRNAs are substantially expressed in embryos rather than adults (Huang et al., 2008; Li et al., 2011; Liang et al., 2007). Using Solexa sequencing, 42 new miRNA candidates were identified in chicken embryos (3, 4, and 5 days old) from 651,273 reads obtained from dissected somite tissue. Eighteen miRNAs were confirmed of which novel miR-10a $(28,660)$ and miR-10b $(113,106)$ were confirmed to have a high number of reads and most abundant respectively indicating that these two miRs are involved in somite development (Rathjen et al., 2009). It was revealed that from stage 
14 of chicken embryo incubation, miR-1 was identified in the somatic myotome which matches with the beginning of skeletal muscle differentiation (Hamburger and Hamilton, 1951); miR-133 from (at stage 15) and miR-206 (at stage 20) were identified in the myocardium and myotome, and somites respectively for fast development of limbs (Darnell et al., 2006). Jebessa et al. (2017) identified several miRNAs in the embryonic stages (E11, E16, and P1). Respectively, 214, 225 and 255 novel miRNAs from 390, 418 and 375 known miRNAs were characterized in chicken skeletal muscle development (Jebessa et al., 2017). The most relatively expressed miRNAs in all the periods were miR-10b-5p, miR-148a-3p, miR-22-3p and miR-133a-3p whereas miR-133a/b, miR-206, miR-486, miR-26a, miR-27b, miR-378, miR-148a, and miR181 (muscle-specific) which play substantial regulatory roles in skeletal muscle and metabolism were extremely present in skeletal muscle (Jebessa et al., 2017; McCarthy and Esser, 2006). In broilers and layers, miR-125b, miR-221, and miR-206 expressions were confirmed in three developmental stages (9e, 17e, and 21a) (Andreote et al., 2014). Each miRNA exhibited a specific expression pattern during muscle development, but the general model of all miRNAs showed similar expression between the two chicken lines. MiR-125b, miR-221, and miR-206 had the same expression levels at stage 9e; miR-125b and miR-206 had a higher expression at stage $17 \mathrm{e}$ than $9 \mathrm{e}$ whereas miR-221 had lower expression. The expression level of miR-125b was maintained at stage 21 a similar to that observed at $17 \mathrm{e}$, and miR-221 expression was again reduced, while miR-206 resumed to the expression level that was observed at 9e (Andreote et al., 2014).

The miRNA transcriptome of broilers and layers revealed 15 differentially expressed miRNAs involved in skeletal muscle growth of which nine were shown in the broilers and six expressed in females (Li et al., 2011). Also, a study in the breast muscle of pedigree male (PeM) broiler and Barred Plymouth Rock (BPR) chicken lines revealed 38 abundantly expressed mature miRNAs (Khatri et al., 2018). In the Pekin duck, a study identified and validated 15 miRNA using qRT-PCR analysis. By comparing the expression profiles among tissues, three muscle-specific miRNAs $(-1,-133$, and -206) were highly expressed in the leg, breast and heart muscles; six (6) were related in myogenesis (miR-103a-3p, miR-107, miR-26a-5p, miR-10a5p, miR-181a-3p and miR-222a); two (2) miRNA (miR-152 and miR-143) were found in all tissues while the remaining four (4) miRNAs (let-7i, miR-23a, miR-24, and miR-214) could be found in either liver or kidney ( $\mathrm{Gu}$ et al., 2014). In quails, 32 miRNAs were expressed at different periods of the early embryo development. After 24 and $30 \mathrm{~h}$ of incubation, expression pattern of 14 of the miRNAs which included let-7a, let-7b, mir-24-3p, mir-26a, mir-122, mir-126, mir-10b decreased in numbers whereas mir-10b, mir-20, mir-103, and mir-196a increased numerically. Finally, the qPCR analysis revealed that seven miRNA: let-7a, let-7b, mir-10b, mir26a, mir-122, mir-125b, and mir-126 were differentially expressed during early quail embryo development (Schellander et al., 2013). 


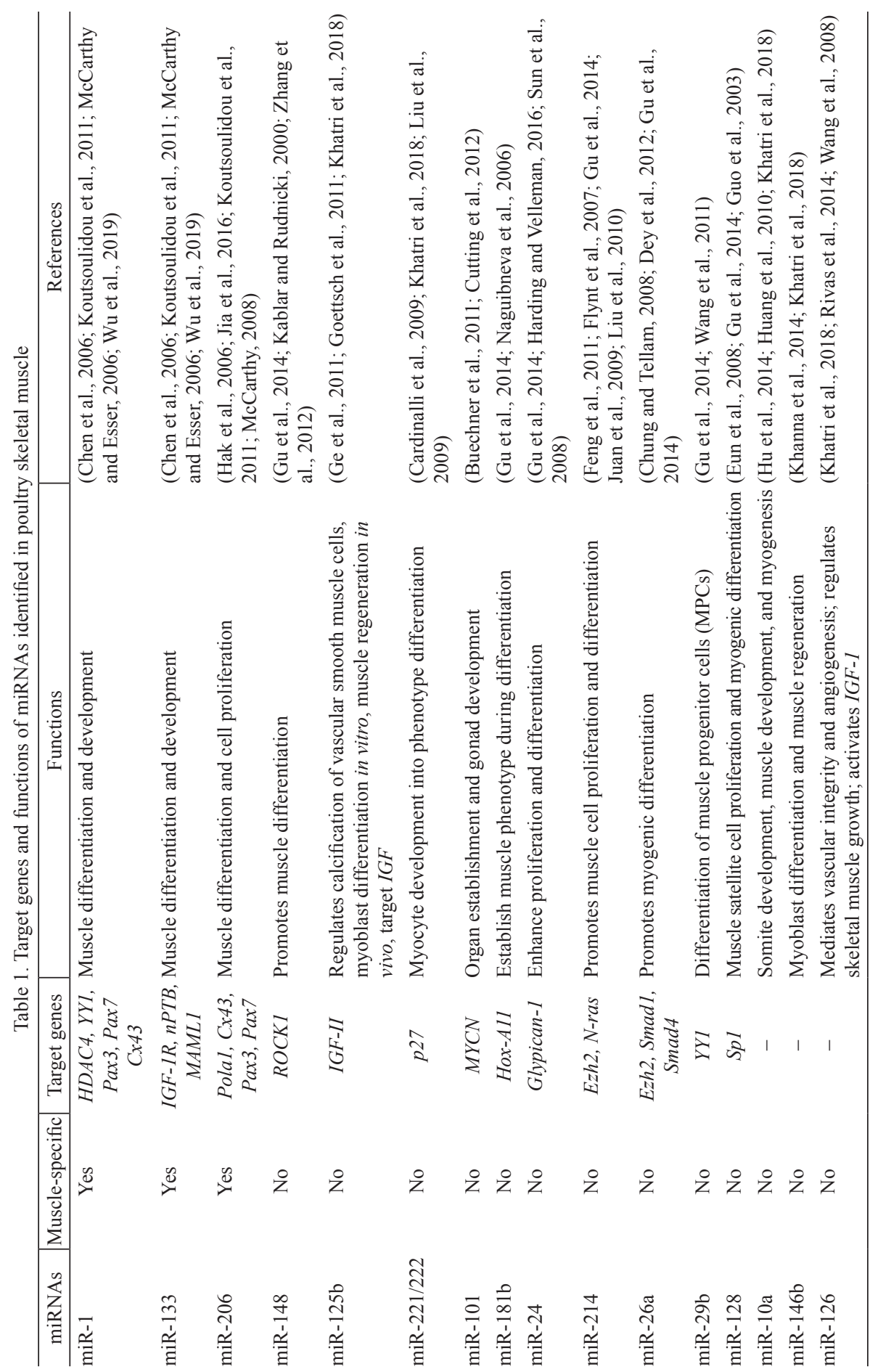




\section{Classification of miRNAs}

All the identified miRNAs can be classified as muscle-specific (myomiRs), and non-muscle specific (non-myomiRs) and each has a particular role it plays in the skeletal muscle development process (Table 1). To study and understand miRNAs in proliferation and differentiation, the $\mathrm{C} 2 \mathrm{C} 12$ mouse myogenic cell line model which mimics several conditions that occur in vivo in skeletal muscle development was used (Burattini et al., 2004). Earlier studies in vitro have established that, in the presence of myogenin, myosin, differentiation genes and under low serum conditions, culture of $\mathrm{C} 2 \mathrm{C} 12$ myoblast could effectuate myoblast to differentiate to form myotubes (Yaffe and Saxel, 1977). However, this C2C12 mainly affected the expression of miRNA in the cell such that seventy-seven miRNAs were up-regulated whereas sixty-eight were down-regulated in differentiation and proliferation stages (Lu et al., 2012). Studies have confirmed that miR-1, miR-133, and miR-206 which were most expressively up-regulated were exceptionally involved in differentiation of skeletal muscle (Chen et al., 2006; Hak et al., 2006). Except for miR-699a which was found to impede muscle differentiation, miR-9-2, miR-122a, miR-703, and miR-805 which were highly down-regulated were seldom found to involve in muscle differentiation (Crippa et al., 2011).

\section{Muscle-specific miRNAs (myomiRs)}

As discovered in humans and mouse, this class of miRNAs has also been implicated in the skeletal muscle development in poultry (Gu et al., 2014; Jebessa et al., 2017; Khatri et al., 2018; Wu et al., 2019). MyomiRs are the miRNAs that solely express in striated muscle and presently comprise miR-1, miR-133, miR-206, miR208a, miR-208b, miR486 and miR-499 (McCarthy, 2008; O'Rourke et al., 2010; van Rooij et al., 2007). However, only three of them (mir-1, mir-133, and mir-206) have been severally studied, recognized and proven in poultry (Jebessa et al., 2017; Wu et al., 2019). Except for miR-206 which is only specific in skeletal-muscle, the other myomiRs express in both skeletal and cardiac muscles (Lagos-Quintana et al., 2002). The expression of the myomiRs is controlled by MRFs (MYOD, MYOG, MRF4, $M Y F 5), M E F 2 s, S R F$ and several transcriptional factors which play crucial roles in each stage of the muscle development process (Braun and Gautel, 2011; Chen et al., 2006; Potthoff and Olson, 2007; Sweetman et al., 2008; Wood et al., 2013; Wu et al., 2019).

\section{Mechanism and functions of miR-1}

MiRNA-1 is associated with the differentiation and development of skeletal muscle. Significantly, researches have proven that miRNA-1 functions in the initial stage through to the later stages in skeletal myocyte development and the homeostatic maintenance of skeletal muscle (Chen et al., 2006). It is also revealed that miR-1 regulates the generation of cardiac muscle and its related diseases (Townley-Tilson et al., 2010). However, these essential functions of miR-1 are regulated and inhibited by several transcription factors. It is revealed that $m T O R$ signaling regulates the expression of miR-1 by controlling $M Y O D$, a transcriptional factor that promotes the expression of miR-1 (Sun et al., 2010). Activation of miR-1 inhibits the expression 
of target genes which suppress muscle development. Histone deacetylase (HDAC4) is a transcriptional repressor which hinders $M E F 2$ protein expression that enhances muscle development (Chen et al., 2006). MiR-1 is capable of constraining the expression of $H D A C 4$ by binding to its $3 \mathrm{UTR}$, thus promoting muscle cell differentiation (Chen et al., 2006; Wu et al., 2019). Also, miR-1 can inhibit the expression of a zincfinger transcription factor, $Y Y 1$ which negatively regulates muscle gene regulation and $P A X 7$ which up-regulates ID2 (inhibitor of DNA binding 2) to repress MYOD regulation (Chen et al., 2010; Lu et al., 2012). More so, miR-1 regulates myogenesis through PI3K/Akt signaling pathway which contributes to muscle growth and hypertrophy (Figure 1) (Bassel-Duby and Olson, 2006; Egerman and Glass, 2014). When Akt phosphorylates, it decreases $\mathrm{FOXO3a}$ (a transcription factor that adversely controls protein synthesis and muscle growth) and regulates miR-1 promoter activity. When the expression of miR-1 decreases, $I G F-1$ and $I G F-1 R$ expression increases because they are target genes to miR-1 (Elia et al., 2009).

\section{Mechanism and functions of miR-133}

MiR-1 and miR-133 have similar regulatory functions and transcription factors because they are all transcribed from the same bicistronic transcripts. miRNA-133 (miR-133a/b) functions early in the myogenic stem cells differentiation into myoblast and the growth of complex muscle tissues (Baquero-Perez et al., 2012; Hak et al., 2006; Koomkrong et al., 2015; Takaya et al., 2009). MiRNA-133 promotes myoblast proliferation and regulates the growth and function of myocardium and bone by inhibiting the activity of SRF and TGFBRI (Wu et al., 2019; Yin et al., 2013; Zhao et al., 2005). It is established that miR-133 repressor decreased the overexpression of miR-133 but greatly increased the expression of $S R F$ and TGFBRI which suggests that overexpression of miR-133 negatively regulates $S R F$ and TGFBRI which control the development of skeletal muscles in ducks (Wu et al., 2019). Also, miR-133 inhibits the expression of $I G F-1 R, n P T B$, and $M A M L 1$. MAML regulates the connection of Notch and $M E F 2$ to arouse muscle differentiation; IGF-IR contributes to embryonic and postnatal development and growth of muscle cell whereas pre-mRNA splicing is controlled by nPTB in muscle and neuron differentiation (Boutz et al., 2007; Huang et al., 2011; Shen et al., 2006). MiR-133a/b regulates MAPK pathway via down-regulation of its transducers (FFGFR1 and PP2AC) to promote differentiation and suppress myoblast proliferation (Feng et al., 2013; Liu et al., 2011). Similar to miR-1, when the expression of myogenin increases, it activates the expression of miR-133 which blocks $I G F-I R$, thereby decreasing the regulation of Akt phosphorylation (Figure 1) (Huang et al., 2011).

\section{Mechanism and functions of miR-206}

As described earlier in the above myomiRs, miR-206 which plays similar roles is also a recognized myogenic miRNA which has significant functions during muscle development, and they are abundantly expressed in the skeletal muscle of many animals including poultry (Liang et al., 2007; McDaneld et al., 2009). However they are not always high because its pattern of expression is temporal. Studies have shown that miR-206 inhibited Pola 1 which is the largest subunit of DNA polymerase A 
(DNA pol a) for DNA synthesis in myoblast differentiation (Hak et al., 2006). The expression of Pax3 and Pax7 (Figure 2) which prevented early differentiation of myoblast is hindered when miR-206 is overexpressed (Chen et al., 2010; Hirai et al., 2010). Overexpression of miR-206 in chicken myoblast increased the expression of myogenin and muscle creatine kinase which are essential genes for muscle differentiation (Jia et al., 2016). A study reported that in the P38 MAPK, ERK1/2, PI3K, and insulin signaling pathways, ANXA2, TPM3, and EIF2AK3 genes are up-regulated when miR-206 is suppressed (Khatri et al., 2018). It is established that ANXA2 (annexin A2) regulates proliferation, migration and cytoskeletal formation in muscle cells (Chen et al., 2014; Draeger et al., 2002); TPM3 (tropomyosin 3) links with actin filament in muscle cells and troponin complex to regulate contraction of the striated muscle in vertebrates (Lawlor et al., 2010) and EIF2AK3 (eukaryotic translation initiation factor 2 alpha kinase 3 ) controls mitochondrial morphology and function (De Mario et al., 2017). Khatri and colleagues proposed that the quick myogenesis displayed in the breast muscle of PeM chickens was due to miR-206 and its own targets interaction (Khatri et al., 2018).

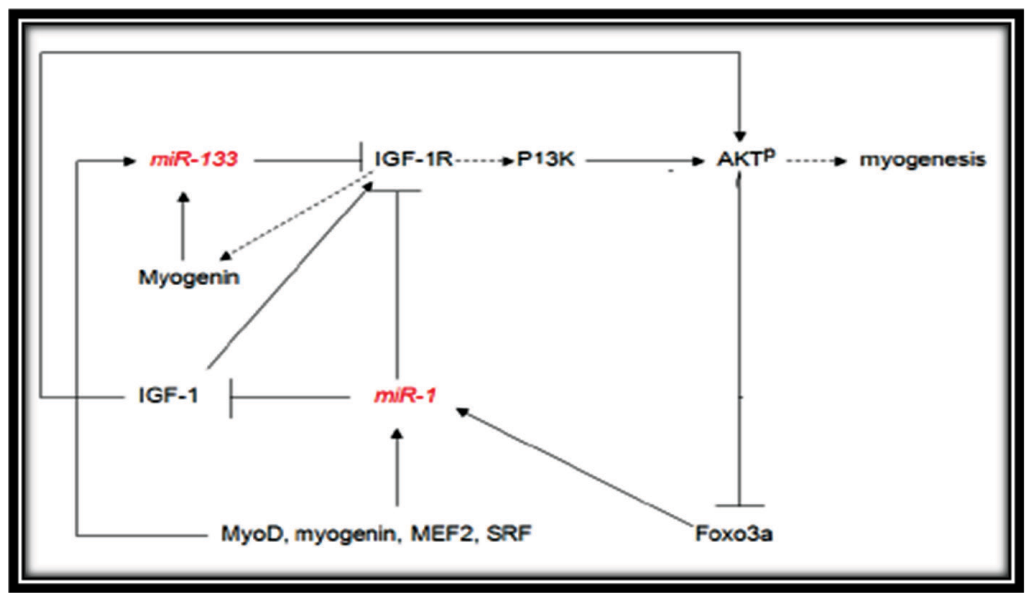

Figure 1. MicroRNAs regulating myogenesis via PI3K/Akt signaling. The dash arrows show the pathway not verified. MYOD (myogenic differentiation); MEF2 (myocyte enhancer factor 2); SRF (serum response factor); AKTP (phospho-AKT); Foxola (forkhead box O1); Foxo3a (forkhead box O3); $I G F-1$ (insulin-like growth factor 1); IGF-IR (insulin-like growth factor 1 receptor); PI3K (phosphoinositide-3-kinase) (Xu et al., 2013 b)

\section{Non-muscle-specific miRNAs (Non-myomiRs)}

Apart from the muscle-specific identified in poultry skeletal muscle, other miRNAs which are non-muscle specific have also been discovered and proven to play vital roles in the muscle development process. Some of the non-myomiRs identified in the poultry skeletal muscle include: let 7, miR-10, miR-21, miR-22-3p, miR-24, miR-26, miR-27, miR-107, miR-125, miR-126, miR-148, miR-181, miR-196, miR221 , miR-222, and miR-214. Some of the miRNAs promote muscle proliferation and differentiation whereas others negatively regulate muscle differentiation. 


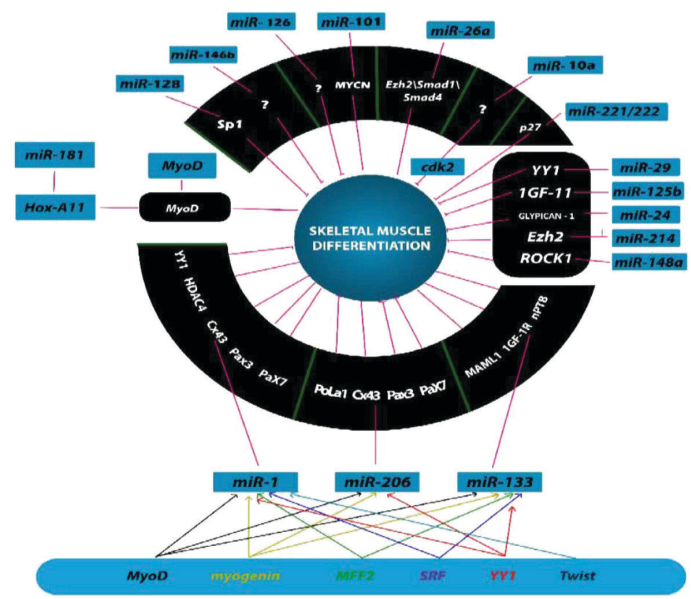

Figure 2. miRNAs regulation in skeletal muscle differentiation in poultry. The figure shows miRNAs (in blue) associated with skeletal muscle differentiation and their mRNA targets (in the black box) and some transcription factors (in the blue box found at the bottom) that control the expression of three musclespecific miRNAs

\section{Mechanism of non-myomiRs that promote muscle proliferation and differ- entiation}

Among the miRNAs discovered in the poultry, miR-24, miR-26a, miR-181, miR-214, and miR-148 are proven to enhance skeletal muscle differentiation. The overexpression of these miRNAs increases the appearance of the MRFs to enhance myoblast differentiation. In $\mathrm{C} 2 \mathrm{C} 12$ myoblast differentiation, the levels of miR-24, miR-26a and miR-148a were expressly up-regulated compared to undifferentiated cells. This implies that overexpression of the mentioned miRNAs enhances myogenic differentiation. The high levels of miR-24, miR-26a and miR-148a, could directly inhibit the appearance of their target genes $T G F-\beta 1, E z h 2$ and ROCK1 respectively which suppress muscle differentiation (Dey et al., 2012; Sun et al., 2008; Zhang et al., 2012). A study revealed that the expression of glypican-1, a target gene of miR24 increased significantly at 24 hours then declined at 72 hours of muscle proliferation and differentiation when miR-24 was inhibited (Harding and Velleman, 2016). Creatine kinase, an enzyme which increases during myogenesis was induced when miR-26a was highly expressed (Chung and Tellam, 2008). The muscle phenotype is established upon the up-regulation of miR-181 during myogenic differentiation by inhibiting the expression of HoxA11, a target gene that represses the differentiation processes (Naguibneva et al., 2006). In the embryonic stem cells, miR-214 increases muscle cells differentiation and stimulates the transcription of developmental regulators by downregulating PcG proteins which repress this progress in the embryonic stem cells. MiR-214 controls the PcG proteins by targeting the Ezh2 3'UTR during transcription (Juan et al., 2009). It also down-regulates N-ras level (proto-oncogene) which inhibits muscle differentiation (Liu et al., 2010). Again, miR-214 promotes 
muscle proliferation. To prove this, $\mathrm{C} 2 \mathrm{C} 12$ cells were transfected with miR-214 inhibitor and cultured in a growth medium which hindered myoblast proliferation. This shows that miR-214 promotes both muscle differentiation and proliferation (Feng et al., 2011). Overexpression of miR-128 inhibited Sp1 expression, an activator of MyoD which regulates cell proliferation and differentiation. MiR-128 was expressively increased in the brain and skeletal muscles (Guo et al., 2003).

\section{Mechanism of non-myomiRs that negatively regulate muscle differentiation}

MiR-221/222 and miR-125 are examples of identified miRNAs in poultry which have been reported to regulate myogenic differentiation negatively (Cardinalli et al., 2009; Ge et al., 2011). A study reported after Northern blot analysis that miR$221 / 222$ acts contrary to miR-133 because they were highly expressed in myoblast to inhibit proliferation and differentiation (Cardinalli et al., 2009). Ras-MAPK signaling pathway down-regulates the expression of miR-221/222 during myogenesis in poultry and mammals (Cardinalli et al., 2009). Even though myoblast proliferation was not altered when miR-125 levels declined, differentiation was negatively regulated in $\mathrm{C} 2 \mathrm{C} 12$ myoblast differentiation. MiR-125 targeted $I G F-I I$ which importantly regulates skeletal myogenesis in the myocytes (Ge et al., 2011).

\section{Conclusion}

Skeletal muscle development is a multi-step process regulated by several genetic factors of which miRNAs play an essential role. Two most important transcription factors; the muscle regulatory factors $(M R F)$ and myocyte enhancer factor $2(M E F 2)$ families help in the formation and differentiation of muscles. The discovery of miRNAs enhances our understanding of the regulatory roles and mechanisms of these miRNAs which are essential for the skeletal muscle differentiation. These miRNAs can be muscle-specific (miR-1, miR-133, and miR-206) and non-muscle specific (miR-24, miR-181, miR-128, and others). The vital functions of miR-1, miR-133 and miR-206 in myogenesis aid us to comprehend the significance of tissue-specific miRNAs. However, the regulatory functions of non-myomiRs in myogenesis cannot be overlooked. Each of the muscle-specific miRNAs has their mRNA target: miR-1 targets and inhibits HDAC4, YY1, Pax 3 and Pax 7; miR-133 targets MAML1, $I G F-I R, n P T B$; and miR-206 targets Pola 1, Pax3, Pax 7. Little is known about the target genes of the other miRNAs. It is projected that several miRNAs control about one-third of mammalian and poultry genes, but the specific targets and roles of most miRNAs have not been discovered which might be helpful in poultry breeding industry.

\section{Acknowledgements}

The authors are thankful to the Science and Technology Plan Project of Guangdong Province (2017A020208066) and Modern Agriculture Key Project of Zhanjiang City (2015A03003) for providing financial support.

\section{Competing Interests}

Conflict of Interest: The authors declare that there is no conflict of interest 


\section{References}

A c c ili D., Arden K.C. (2004). FoxOs at the crossroads of cellular review metabolism, differentiation, and transformation. Cell, 117: 421-426.

And r e o t e A.P.D., R o s a ri o M.F., L e dur M.C., J org e E.C., S o n s t e g a rd T.S., M a t u k u m a $11 \mathrm{i}$ L., C ou t in ho L.L. (2014). Identification and characterization of microRNAs expressed in chicken skeletal muscle. Genet. Mol. Res., 13: 1465-1479; https://doi.org/10.4238/2014.March.6.5.

B a quero-Perez B., Kuchipudi S.V., Nelli R.K., Chang K.C. (2012). A simplified but robust method for the isolation of avian and mammalian muscle satellite cells. BMC Cell Biol. 13, 16; https://doi.org/10.1186/1471-2121-13-16.

B a s s e l- D u by R., O ls on E.N. (2006). Signaling pathways in skeletal muscle remodeling. Annu. Rev. Biochem.,75: 19-37; https://doi.org/10.1146/annurev.biochem.75.103004.142622.

B e r k e s C.A., Ta p s c ot t S.J. (2005). MyoD and the transcriptional control of myogenesis. Semin. Cell Dev. Biol., 16: 585-595; https://doi.org/10.1016/j.semcdb.2005.07.006.

B o d in e S.C., S tit t T.N., Gonzalez M., K 1 in e W.O., S to ver G.L., B a u e r l e in R., Zlot chenko E., Scrimgeour A., Law rence J.C., Glass D.J., Yancopoulos G.D. (2001). Akt/ mTOR pathway is a crucial regulator of skeletal muscle hypertrophy and can prevent muscle atrophy in vivo. Nat. Cell Biol., 3: 1014-1019; https://doi.org/10.1038/ncb1101-1014.

B outz P.L., Chaw la G., S to ilov P., B lack D.L. (2007). MicroRNAs regulate the expression of the alternative splicing factor nPTB during muscle development. Genes Dev., 21: 71-84; https:// doi.org/10.1101/gad.1500707.

B r a u n T., G a u t e 1 M. (2011). Transcriptional mechanisms regulating skeletal muscle differentiation, growth and homeostasis. Nat. Rev. Mol. Cell Biol., 12: 349-361; https://doi.org/10.1038/nrm3118.

B u ck ing ha m M. (2006). Myogenic progenitor cells and skeletal myogenesis in vertebrates. Curr. Opin. Genet. Dev.; https://doi.org/10.1016/j.gde.2006.08.008.

B u e chner J., Tø m te E., H a u g B.H., Hen riks e n J.R., Løk k e C., F 1 æg s ta d T., E invik C. (2011). Tumour-suppressor microRNAs let-7 and mir-101 target the proto-oncogene MYCN and inhibit cell proliferation in MYCN-amplified neuroblastoma. Br. J. Cancer, 105: 296-303; https:// doi.org/10.1038/bjc.2011.220.

Burattini S., Ferri R., Battistelli M., Curci R., Luchetti F., Falcieri E. (2004). $\mathrm{C} 2 \mathrm{C} 12$ murine myoblasts as a model of skeletal muscle development: Morpho-functional characterization. Eur. J. Histochem., 48: 223-233.

Cardinalli B., Castella ni L., Fas a naro P., B a s s o A., A le mà S., Martelli F., Fal c o n e G. (2009). Microrna-221 and microrna-222 modulate differentiation and maturation of skeletal muscle cells. PLoS One, 4; https://doi.org/10.1371/journal.pone.0007607.

Castigliego L., Armani A., Grifoni G., Rosati R., Mazzi M., Gianfaldoni D., Guidi A. (2010). Effects of growth hormone treatment on the expression of somatotropic axis genes in the skeletal muscle of lactating Holstein cows. Domest. Anim. Endocrinol., 39: 40-53; https://doi.org/10.1016/j.domaniend.2010.02.001

Chen B., X u J., H e X., X u H., Li G., D u H., N i e Q., Z hang X. (2015). A genome-wide mRNA screen and functional analysis reveal FOXO3 as a candidate gene for chicken growth. PLoS One, 10: 1-22; https://doi.org/10.1371/journal.pone.0137087.

Chen J.F., Mandel E.M., Thom s on J.M., Wu Q., Calli s T.E., Hammond S.M., Con lo $\mathrm{n}$ F.L., Wang D.Z. (2006). The role of microRNA-1 and microRNA-133 in skeletal muscle proliferation and differentiation. Nat. Genet., 38: 228-233; https://doi.org/10.1038/ng1725.

Chen J.F., Ta o Y., Li J., D eng Z., Yan Z., Xi a o X., Wang D.Z. (2010) microRNA-1 and microRNA-206 regulate skeletal muscle satellite cell proliferation and differentiation by repressing Pax7. J. Cell Biol., 190: 867-879; https://doi.org/10.1083/jcb.200911036.

Chen L., Li Y.S., C u i J., N ing J.N., Wang G.S., Q i an G.S., L u K.Z., Y i B. (2014). MiR-206 controls the phenotypic modulation of pulmonary arterial smooth muscle cells induced by serum from rats with Hepatopulmonary syndrome by regulating the target gene, Annexin A2. Cell. Physiol. Biochem., 34: 1768-1779; https://doi.org/10.1159/000366377.

Chung F.W., Tella m R.L. (2008). MicroRNA-26a targets the histone methyltransferase enhancer of zeste homolog 2 during myogenesis. J. Biol. Chem., 283: 9836-9843; https://doi.org/10.1074/ jbc.M709614200. 
Clop A., Marcq F., Takeda H., Pirottin D., Tordoir X., Bibé B., Bouix J., Caiment F., Els en J.M., Eychenne F., Larzul C., Laville E., Me ish F., Milenkovic D., Tobin J., Charlier C., G e orges M. (2006). A mutation creating a potential illegitimate microRNA target site in the myostatin gene affects muscularity in sheep. Nat. Genet., 38: 813-818; https://doi.org/10.1038/ng1810.

Crippa S., Cass ano M., Mess ina G., Galli D., Galvez B.G., Curk T., Altomare C., Ronzoni F., Toelen J., Gij sbers R., Debyser Z., Jans sens S., Zu pan B., Zaza A., Cossu G., Sampa olesi M. (2011). miR669a and miR669q prevent skeletal muscle differentiation in postnatal cardiac progenitors. J. Cell Biol., 193: 1197-1212; https://doi.org/10.1083/ jcb.201011099.

Crist C.G., Montarras D., Pallafacchina G., Rocancourt D., Cumano A., Conw a y S.J., B u c king ham M. (2009). Muscle stem cell behavior is modified by microRNA-27 regulation of Pax3 expression. Proc. Natl. Acad. Sci., 106: 13383-13387; https://doi.org/10.1073/ pnas.0900210106.

Cui T.X., Schwartz J., Piwien-Pilipuk G., Lanning N., Rathore M., LaPens e e C.R., Calines cu A.-A., Lin G., Jin H., Q in Z.S., Carter-Su C., Streeter C. (2011). C/EBP $\beta$ mediates growth hormone-regulated expression of multiple target genes. Mol. Endocrinol., 25: 681-693; https://doi.org/10.1210/me.2010-0232.

Cutting A.D., B annister S.C., Doran T.J., S in c la ir A.H., Tizard M.V.L., S m ith C.A. (2012). The potential role of microRNAs in regulating gonadal sex differentiation in the chicken embryo. Chromosom. Res., 20: 201-213; https://doi.org/10.1007/s10577-011-9263-y.

Darnell D.K., Ka ur S., S t an is law S., Kon i e c zka J.K., Yats ki ev y ch T.A., Ant in P.B. (2006). MicroRNA expression during chick embryo development. Dev. Dyn., 235: 3156-3165; https://doi.org/10.1002/dvdy.20956.

De Mario A., Quintana-Cabrera R., Martinvalet D., Giacomello M. (2017). (Neuro)degenerated Mitochondria-ER contacts. Biochem. Biophys. Res. Commun., 483: 1096-1109; https://doi.org/10.1016/j.bbrc.2016.07.056.

D e y B.K., Ga ga n J., Ya n Z., D u t t a A. (2012). miR-26a is required for skeletal muscle differentiation and regeneration in mice. Genes Dev., 26: 2180-2191; https://doi.org/10.1101/gad.198085. 112.

Draeger A., B abiy chuk E.B., S challer J., Palstra R.-J.T.S., Kämpfer U. (2002). Annexin VI participates in the formation of a reversible, membrane-cytoskeleton complex in smooth muscle cells. J. Biol. Chem., 274: 35191-3519; https://doi.org/10.1074/jbc.274.49.35191.

Dupont J., Holzenberger M. (2003). Biology of insulin-like growth factors in development. Birth Defects Res. Part C: Embryo Today: Rev.; https://doi.org/10.1002/bdrc.10022.

E g e r m a n M.A., G l a s s D.J. (2014). Signaling pathways controlling skeletal muscle mass. Crit. Rev. Biochem. Mol. Biol., 49: 59-68; https://doi.org/10.3109/10409238.2013.857291.

Elia L., Contu R., Quintavalle M., Varrone F., Chimenti C., Russo M.A., Cimino V., De Marinis L., Frustaci A., Catalucci D., Condorelli G. (2009). Reciprocal regulation of microRNA-1 and insulin-like growth factor-1 signal transduction cascade in cardiac and skeletal muscle in physiological and pathological conditions. Circulation, 120: 2377-2385; https://doi.org/10.1161/CIRCULATIONAHA.109.879429.

Eu n J.L., B a e k M., G u s e v Y., B r a c k e t t D.J., N u ov o G.J., S c h m i t tge n T.D. (2008). Systematic evaluation of microRNA processing patterns in tissues, cell lines, and tumors. RNA, 14: 35-42; https://doi.org/10.1261/rna.804508.

F eng Y., C a o J.H., Li X.Y., Z ha o S.H. (2011). Inhibition of miR-214 expression represses proliferation and differentiation of $\mathrm{C} 2 \mathrm{C} 12$ myoblasts. Cell Biochem. Funct., 29: 378-383; https://doi. org/10.1002/cbf.1760.

F eng Y., Niu L.L., We i W., Zhang W.Y., Li X.Y., C a o J.H., Z ha o S.H. (2013). A feedback circuit between miR-133 and the ERK1/2 pathway involving an exquisite mechanism for regulating myoblast proliferation and differentiation. Cell Death Dis., 4: 934; https://doi.org/10.1038/cddis.2013.462.

Flynt A.S., Li N., Thatcher E.J., Solnica-Krezel L., Patton J.G. (2007). Zebrafish miR-214 modulates Hedgehog signaling to specify muscle cell fate. Nat. Genet., 39: 259-263; https://doi.org/10.1038/ng1953. 
G a n W., He H., L i L. (2016). Molecular cloning, characterisation and functional analysis of the duck Forkhead box O3 (FOXO3) gene. Br. Poult. Sci., 57: 143-150; https://doi.org/10.1080/00071668. 2015.113550 .

G e Y., S u n Y., Ch en J. (2011). IGF-II is regulated by microRNA-125b in skeletal myogenesis. J. Cell Biol., 192: 69-81; https://doi.org/10.1083/jcb.201007165.

Glazov E.A., Cottee P.A., B arris W.C., Moore R.J., Dalrymple B.P., Tizard M.L. (2008). A microRNA catalog of the developing chicken embryo identified by a deep sequencing approach. Genome Res., 18: 957-964; https://doi.org/10.1101/gr.074740.107.

Goet ts ch C., Rauner M., Pacyna N., Hempel U., Bornstein S.R., Hofbauer L.C. (2011). MiR-125b regulates calcification of vascular smooth muscle cells. Am. J. Pathol., 179: 1594-1600; https://doi.org/10.1016/j.ajpath.2011.06.016.

Grifone R., Demignon J., Giordani J., Niro C., Souil E., Bertin F., Laclef C., $\mathrm{X}$ u P.X., M a ir e P. (2007). Eya1 and Eya2 proteins are required for hypaxial somitic myogenesis in the mouse embryo. Dev. Biol., 302: 602-616; https://doi.org/10.1016/j.ydbio.2006.08.059.

Gu L., X u T., H u a ng W., X i e M., S un S., H o u S. (2014). Identification and profiling of microRNAs in the embryonic breast muscle of Pekin duck. PLoS One, 9: 1-13; https://doi.org/10.1371/ journal.pone.0086150.

$\mathrm{G} \mathrm{u} \mathrm{Z.} \mathrm{(2004).} \mathrm{The} \mathrm{single} \mathrm{nucleotide} \mathrm{polymorphisms} \mathrm{of} \mathrm{the} \mathrm{chicken} \mathrm{myostatin} \mathrm{gene} \mathrm{are} \mathrm{associated} \mathrm{with}$ skeletal muscle and adipose growth. Sci. China Ser. C 47, 25; https://doi.org/10.1360/02yc0201.

Guo C.S., Degnin C., Fiddler T.A., S t a uffer D., Thayer M.J. (2003). Regulation of MyoD activity and muscle cell differentiation by MDM2, pRb, and Sp1. J. Biol. Chem., 278: 22615-22622; https://doi.org/10.1074/jbc.M301943200.

H a c h e R.J.G., W i p e r - B e r g e r o n N., S a le m H.A., W u D., T o m 1 in s o n J.J. (2007). Glucocorticoid-stimulated preadipocyte differentiation is mediated through acetylation of C/EBPbeta by GCN5. Proc. Natl. Acad. Sci., 104: 2703-2708; https://doi.org/10.1073/pnas.0607378104.

Hadjiargyrou M., Lombardo F., Zha o S., Ahrens W., Joo J., Ahn H., Jurman M., W h i t e D.W., R u b i n C.T. (2002). Transcriptional profiling of bone regeneration. Insight into the molecular complexity of wound repair. J. Biol. Chem., 277: 30177-30182; https://doi.org/10.1074/ jbc.M203171200.

Hak K.K., Yong S.L., Sivaprasad U., Malhotra A., Dutta A. (2006). Muscle-specific microRNA miR-206 promotes muscle differentiation. J. Cell Biol., 174: 677-687; https://doi. org/10.1083/jcb.200603008.

H a m burger V., H a mil t o n H.L. (1951). A series of normal stages in the development of the chick embryo. J. Morphol., 88: 49-92; https://doi.org/10.1002/jmor.1050880104.

H a r d ing R.L., Ve 11 e m a n S.G. (2016). MicroRNA regulation of myogenic satellite cell proliferation and differentiation. Mol. Cell. Biochem., 412: 181-195; https://doi.org/10.1007/s11010-0152625-6.

H a r r i s L.K., We s t w o o d M. (2012). Biology and significance of signalling pathways activated by IGF-II. Growth Factors; https://doi.org/10.3109/08977194.2011.640325.

Hennebry A., B erry C., Siriett V., O'Callaghan P., Chau L., Watson T., Sharm a M., K a m b a dur R. (2008). Myostatin regulates fiber-type composition of skeletal muscle by regulating MEF2 and MyoD gene expression. AJP Cell Physiol., 296: 525-534; https://doi. org/10.1152/ajpcell.00259.2007.

H i c k s J.A., T e m b hu rn e P., L i u H.C. (2008). MicroRNA expression in chicken embryos. Poultry Sci., 87: 2335-2343; https://doi.org/10.3382/ps.2008-00114.

H i c k s J.A., Tra k o o lj u 1 N., L i u H.-C. (2010). Discovery of chicken microRNAs associated with lipogenesis and cell proliferation. Physiol. Genomics, 41: 185-193; https://doi.org/10.1152/physiolgenomics.00156.2009.

Hillier L.W., Miller W., B irney E., Warren W., Hardison R.C., Ponting C.P., B ork P., B urt D.W., Groen en M.A.M., D e lan y M.E., D o d g s o n J.B. (2004). Sequence and comparative analysis of the chicken genome provide unique perspectives on vertebrate evolution. Nature, 432: 695-716; https://doi.org/10.1038/nature03154.

Hirai H., Verma M., Watanabe S., Tastad C., As akura Y., Asakura A. (2010). MyoD regulates apoptosis of myoblasts through microRNA-mediated down-regulation of Pax3. J. Cell Biol., 191: 347-365; https://doi.org/10.1083/jcb.201006025. 
Hu R., Pan W., Fedulov A. V., Jester W., Jones M.R., We is s S.T., Panettieri R.A., Tant is ir a K., L u Q. (2014). MicroRNA-10a controls airway smooth muscle cell proliferation via direct targeting of the PI3 kinase pathway. FASEB J., 28: 2347-2357; https://doi.org/10.1096/ fj.13-247247.

Huang H., Xi e C., Sun X., Ritchie R.P., Zhang J., Eugen e Chen Y. (2010). miR-10a contributes to retinoid acid-induced smooth muscle cell differentiation. J. Biol. Chem., 285: 9383-9389; https://doi.org/10.1074/jbc.M109.095612.

Huang M.B., X u H., Xi e S.J., Z hou H., Qu L.H. (2011). Insulin-like growth factor-1 receptor is regulated by microRNA-133 during skeletal myogenesis. PLoS One, 6; https://doi.org/10.1371/ journal.pone.0029173.

H u a n g T.H., Z hu M.J., L i X.Y., Z h a o S.H. (2008). Discovery of porcine microRNAs and profiling from skeletal muscle tissues during development. PLoS One, 3; https://doi.org/10.1371/journal. pone.0003225.

Hunter R.B., Kandarian S.C. (2004). Disruption of either the Nfkb1 or the Bcl3 gene inhibits skeletal muscle atrophy. J. Clin. Invest., 114: 1504-1511; https://doi.org/10.1172/JCI200421696.

Ishibashi J., Perry R.L., Asakura A., Rudnicki M.A. (2005). MyoD induces myogenic differentiation through cooperation of its NH2- and COOH-terminal regions. J. Cell Biol., 171: 471-482; https://doi.org/10.1083/jcb.200502101.

J e b e s s a E., O u y ang H., A b d a 11 a B.A., L i Z. (2017). Characterization of miRNA and their target gene during chicken embryo skeletal muscle development. Oncotarget, 9: 17309-17324; https:// doi.org/10.18632/oncotarget.22457.

J i a X., L in H., A b d a 11 a B.A., N i e Q. (2016). Characterization of miR-206 promoter and its association with birthweight in chicken. Int. J. Mol. Sci. 17, 559; https://doi.org/10.3390/ijms17040559.

J u a n A.H., K u m a r R.M., M a r x J.G., You n g R.A., S a r t o r e 11 i V. (2009). Mir-214-dependent regulation of the polycomb protein Ezh2 in skeletal muscle and embryonic stem cells. Mol. Cell., 36: 61-74; https://doi.org/10.1016/j.molcel.2009.08.008.

Junqing L., Shu is heng H., We i H., Juny ing Y., We nw u W. (2011). Polymorphisms in the myostatin gene and their association with growth and carcass traits in duck. African J. Biotechnol., 10: 11309-11312; https://doi.org/10.5897/AJB11.512.

Kablar B., Rudnicki M.A. (2000). Skeletal muscle development in the mouse embryo. Histol. Histopathol.; https://doi.org/10.14670/HH-15.649.

Khanna N., Ge Y., Chen J. (2014). MicroRNA-146b promotes myogenic differentiation and modulates multiple gene targets in muscle cells. PLoS One, 9; https://doi.org/10.1371/journal. pone. 0100657 .

Khatri B., S e o D., Shous e S., Pan J.H., Hud son N.J., Kim J.K., B ottje W., Kong B.C. (2018). MicroRNA profiling associated with muscle growth in modern broilers compared to an unselected chicken breed. BMC Genomics, 19: 1-10; https://doi.org/10.1186/s12864-018-5061-7.

Koomkrong N., Theerawatanasirikul S., Boonkaewwan C., Jaturasitha S., $\mathrm{K}$ a y a n A. (2015). Breed-related number and size of muscle fibres and their response to carcass quality in chickens. Ital. J. Anim. Sci., 14: 638-642; https://doi.org/10.4081/ijas.2015.4145.

Koutsoulidou A., Mastroyiannopoulos N.P., Furling D., Uney J.B., Phylact o u L.A. (2011). Expression of miR-1, miR-133a, miR-133b and miR-206 increases during development of human skeletal muscle. BMC Dev. Biol.,11: 1-9; https://doi.org/10.1186/1471213X-11-34.

Lagos-Quintana M., Rauhut R., Yalcin A., Meyer J., Lendeckel W., Tuschl T. (2002). Identification of tissue-specific MicroRNAs from mouse. Curr. Biol., 12: 735-739; https:// doi.org/10.1016/S0960-9822(02)00809-6.

Lawlor M.W., DeChene E.T., Roumm E., Geggel A.S., Moghadaszadeh B., B e g g s A.H. (2010). Mutations of tropomyosin 3 (TPM3) are common and associated with type 1 myofiber hypotrophy in congenital fiber type disproportion. Hum. Mutat., 31: 176-183; https://doi. org/10.1002/humu.21157.

Li T., Wu R., Z hang Y., Z hu D. (2011). A systematic analysis of the skeletal muscle miRNA transcriptome of chicken varieties with divergent skeletal muscle growth identifies novel miRNAs and differentially expressed miRNAs. BMC Genomics, 12; https://doi.org/10.1186/1471-2164-12186. 
Li Z., Abdalla B.A., Zheng M., He X., Ca i B., Han P., Ouyang H., Chen B., Nie Q., $\mathrm{Z}$ h a $\mathrm{g}$ X. (2018). Systematic transcriptome-wide analysis of mRNA-miRNA interactions reveals the involvement of miR-142-5p and its target (FOXO3) in skeletal muscle growth in chickens. Mol. Genet. Genomics, 293: 69-80; https://doi.org/10.1007/s00438-017-1364-7.

L i a n g Y., R i d z o n D., Wo n g L., C h e n C. (2007). Characterization of microRNA expression profiles in normal human tissues. BMC Genomics, 8: 166; https://doi.org/10.1186/1471-2164-8-166.

Liu C., Gersch R.P., Hawke T.J., Hadjiargyrou M. (2010). Silencing of Mustn1 inhibits myogenic fusion and differentiation. Am. J. Physiol. Physiol., 298: 1100-1108; https://doi. org/10.1152/ajpcell.00553.2009.

Li u J., Luo X.J., Xiong A.W., Zhang Z., Di Yue S., Zhu M.S., Cheng S.Y. (2010). MicroRNA-214 promotes myogenic differentiation by facilitating exit from mitosis via down-regulation of proto-oncogene N-ras. J. Biol. Chem.; https://doi.org/10.1074/jbc.M110.115824.

Liu N., Bezprozvannaya S., Shelton J.M., Frisard M.I., Hulver M.W., Mc Milla n R.P., Wu Y., Vo e 1 ke r K.A., Grange R.W., Rich ard so n J.A., B a s se l- D uby R., O 1 s o n E.N. (2011). Mice lacking microRNA 133a develop dynamin 2-dependent centronuclear myopathy. J. Clin. Invest., 121: 3258-3268; https://doi.org/10.1172/JCI46267.

L i u X., Ch en g Y., Z h ang S., L in Y., Yang J., Z h a n g r C. (2009). A necessary role of miR-221 and miR-222 in vascular smooth muscle cell prolife ation and neointimal hyperplasia. Circ. Res., 104: 476-486; https://doi.org/10.1161/CIRCRESAHA.108.185363.

L u L., Zhou L., Che n E.Z., S un K., Ji ang P., Wang L., S u X., S un H., Wang H. (2012). A novel YY1-miR-1 regulatory circuit in skeletal myogenesis revealed by genome-wide prediction of YY1-miRNA network. PLoS One 7. https://doi.org/10.1371/journal.pone.0027596.

L u o W., N i e Q., Z h ang X. (2013). MicroRNAs involved in skeletal muscle differentiation. J. Genet. Genomics, 40: 107-116; https://doi.org/10.1016/j.jgg.2013.02.002.

M c Carth y J.J. (2008). MicroRNA-206: The skeletal muscle-specific myomiR. Biochim. Biophys. Acta - Gene Regul. Mech., 1779: 682-691; https://doi.org/10.1016/j.bbagrm.2008.03.001.

M c C a r th y J.J., E s s e r K.A. (2006). MicroRNA-1 and microRNA-133a expression are decreased during skeletal muscle hypertrophy. J. Appl. Physiol., 102: 306-313; https://doi.org/10.1152/japplphysiol.00932.2006.

McDaneld T.G., Smith T.P.L., Doumit M.E., Miles J.R., Coutinho L.L., Sonstegard T.S., Matukumalli L.K., N on nem an D.J., Wied mann R.T. (2009). MicroRNA transcriptome profiles during swine skeletal muscle development. BMC Genomics, 10: 77; https:// doi.org/10.1186/1471-2164-10-77.

Mendias C.L., B akhurin K.I., Faulkner J.A. (2008). Tendons of myostatin-deficient mice are small, brittle, and hypocellular. Proc. Natl. Acad. Sci., 105: 388-393; https://doi.org/10.1073/ pnas. 0707069105 .

M o s s F.P., L e blond C.P. (1971). Satellite cells as the source of nuclei in muscles of growing rats. Anat. Rec., 170: 421-435; https://doi.org/10.1002/ar.1091700405.

N a gu ibneva I., A m e y ar-Zazou a M., Polesskaya A., A it - S i- Ali S., Gro is man R., S ou idi M., Cuvellier S., H a rel-Bellan A. (2006). The microRNA miR-181 targets the homeobox protein Hox-A11 during mammalian myoblast differentiation. Nat. Cell Biol., 8: 278284; https://doi.org/10.1038/ncb1373.

O 'R o urk e J.R., M c A n ally J., More si V., G erard R.D., S u the r 1 and L.B., O 1 s on E.N., Richardson J.A., Small E.M. (2010). Regulation of PI3-kinase/Akt signaling by muscle-enriched microRNA-486. Proc. Natl. Acad. Sci., 107: 4218-4223; https://doi.org/10.1073/ pnas. 1000300107.

P o t th off M.J., O 1 s o n E.N. (2007). MEF2: a central regulator of diverse developmental programs. Development, 134: 4131-4140; https://doi.org/10.1242/dev.008367.

Rathjen T., Pais H., S we et man D., Moult on V., Munsterberg A., Dalmay T. (2009). High throughput sequencing of microRNAs in chicken somites. FEBS Lett., 583: 1422-1426; https://doi.org/10.1016/j.febslet.2009.03.048.

Richards M.P., P och S.M., M c Murtry J.P. (2005). Expression of insulin-like growth factor system genes in liver and brain tissue during embryonic and post-hatch development of the turkey. Comp. Biochem. Physiol. - A Mol. Integr. Physiol., 141: 76-86; https://doi.org/10.1016/j. cbpb.2005.04.006. 
Rivas D.A., Le s s ard S.J., Rice N.P., Lustgarten M.S., S o K., Good ye a r L.J., P a rn e 11 L.D., F i e $1 \mathrm{~d}$ in g R.A. (2014). Diminished skeletal muscle microRNA expression with aging is associated with attenuated muscle plasticity and inhibition of IGF-1 signaling. FASEB J., 28: 4133-4147; https://doi.org/10.1096/fj.14-254490.

S a c c one V., Puri P.L. (2010). Epigenetic regulation of skeletal myogenesis. Organogenesis, 6: 48-53; https://doi.org/10.4161/org.6.1.11293.

Schellander K., Holker M., Hossain M.M., Tesfaye D., Salilew-Wondim D., Cinar M.U., Kocamis H., Mohammadi-S ang cheshmeh A. (2013). Expression of microRNA and microRNA processing machinery genes during early quail (Coturnix japonica) embryo development. Poultry Sci., 92: 787-797; https://doi.org/10.3382/ps.2012-02691.

Shen H., McElhinny A.S., Ca o Y., Gao P., Liu J., Bronson R., Griffin J.D., Wu L. (2006). The Notch coactivator, MAML1, functions as a novel coactivator for MEF2C-mediated transcription and is required for normal myogenesis. Genes Dev., 20: 675-688; https://doi.org/10.1101/ gad.1383706.

S ong C.L., Li u H.H., K o u J., L v L., Li L., Wang W.X., Wang J.W. (2012). Expression profile of insulin-like growth factor system genes in muscle tissues during the postnatal development growth stage in ducks. Genet. Mol. Res., 12: 4500-4514; https://doi.org/10.4238/2013.May.6.3.

S u m a riw a 11 a V.M., K le in W.H. (2001). Similar myogenic functions for myogenin and MRF4 but not MyoD in differentiated murine embryonic stem cells. Genesis, 30: 239-249; https://oi. org/10.1002/gene.1070.

Sun Q., Zhang Y., Yang G., Chen X., Zhang Y., Cao G., Wang J., Sun Y., Zhang P., F a n M., S h a o N., Yang X. (2008). Transforming growth factor- $\beta$-regulated miR-24 promotes skeletal muscle differentiation. Nucleic Acids Res., 36: 2690-2699; https://doi.org/10.1093/nar/gkn032.

S un Y., G e Y., D rn e vi c h J., Z h a o Y., B a n d M., C h e n J. (2010). Mammalian target of rapamycin regulates miRNA-1 and follistatin in skeletal myogenesis. J. Cell Biol., 189: 1157-1169; https:// doi.org/10.1083/jcb.200912093.

Swe etman D., Goljanek K., Rathjen T., Oustanina S., Braun T., Dalmay T., Münsterberg A. (2008). Specific requirements of MRFs for the expression of muscle specific microRNAs, miR-1, miR-206 and miR-133. Dev. Biol., 321: 491-499; https://doi.org/10.1016/j.ydbio.2008.06.019.

Takay a T., Ono K., Kaw a mura T., Takanabe R., Kaichi S., Morimoto T., Wada H., K it a T., Sh im ats u A., H a s e g a w a K. (2009). MicroRNA-1 and MicroRNA-133 in spontaneous myocardial differentiation of mouse embryonic stem cells. Circ. J., 73: 1492-1497; https:// doi.org/10.1253/circj.CJ-08-1032.

Townley-Tils on W.H.D., Call is T.E., Wang D. (2010). MicroRNAs 1, 133, and 206: Critical factors of skeletal and cardiac muscle development, function, and disease. Int. J. Biochem. Cell Biol.; https://doi.org/10.1016/j.biocel.2009.03.002.

van der Horst A., Burgering B.M.T., (2007). Stressing the role of FoxO proteins in lifespan and disease. Nat. Rev. Mol. Cell Biol., 8: 440-450; https://doi.org/10.1038/nrm2190.

van Rooij E., Sutherland L.B., Qi X., Richardson J.A., Hill J., Olson E.N. (2007). Control of stress-dependent cardiac growth and gene expression by a microRNA. Science, 80: 575-579; https://doi.org/10.1126/science.1139089.

van Rooij E., Liu N., Ols on E.N. (2008). MicroRNAs flex their muscles. Trends Genet., 24: 159-166; https://doi.org/10.1016/j.tig.2008.01.007.

Vell e m a n S.G., N e s tor K.E., C oy C.S., H a r ford I., A n th on y N.B. (2010). Effect of posthatch feed restriction on broiler breast muscle development and muscle transcriptional regulatory factor gene and heparan sulfate proteoglycan expression. Int. J. Poult. Sci., 9: 417-425; https://doi. org/10.3923/ijps.2010.417.425.

Wang H., Li X., Li u H., S un L., Zhang R., Li L., Wang ding M., Wang J. (2016). Six1 induces protein synthesis signaling expression in duck myoblasts mainly via up-regulation of mTOR. Genet. Mol. Biol., 39: 151-161; https://doi.org/10.1590/1678-4685-GMB-2015-0075.

Wang S., A urora A.B., J ohn son B.A., Q i X., M c A nally J., Hi 11 J.A., Ri c hard son J.A., B a s s el-Duby R., Ols on E.N. (2008). The endothelial-specific MicroRNA miR-126 governs vascular integrity and angiogenesis. Dev. Cell, 15: 261-271; https://doi.org/10.1016/j.devcel.2008.07.002. 
Wang X.H., Hu Z., Kle in J.D., Zhang L., Fang F., Mitch W.E. (2011). Decreased miR29 suppresses myogenesis in CKD. J. Am. Soc. Nephrol., 22: 2068-2076; https://doi.org/10.1681/ ASN.2010121278.

W h i te R.B., B i ér i n x A., G n o c c h i V.F., Z a m m it P.S. (2010). Dynamics of muscle fibre growth during postnatal mouse development, BMC Developmental Biology, 10.

W o o d W.M., E t e m a d S., Y a m a m o t o M., G o ld h a m e r D.J. (2013). MyoD-expressing progenitors are essential for skeletal myogenesis and satellite cell development. Dev. Biol., 384: 114-127; https://doi.org/10.1016/j.ydbio.2013.09.012.

Wu N., Gu T., Lu L., Ca o Z., S ong Q., Wang Z., Zhang Y., Chang G., Xu Q., Chen G. (2019). Roles of miRNA-1 and miRNA-133 in the proliferation and differentiation of myoblasts in duck skeletal muscle. J. Cell. Physiol., 234: 3490-3499; https://doi.org/10.1002/jcp.26857.

X u T., Huang W., Zh ang X., Ye B., Z hou H., H ou S. (2012). Identification and characterization of genes related to the development of breast muscles in Pekin duck. Mol. Biol. Rep., 39: 7647-7655; https://doi.org/10.1007/s11033-012-1599-7.

X u T.S., G u L.H., Z h ang X.H., Ye B.G., L i u X.L., H o u S.S. (2013 a). Characterization of myostatin gene (MSTN) of Pekin duck and the association of its polymorphism with breast muscle traits. Genet. Mol. Res., 12: 3166-3177; https://doi.org/10.4238/2013.February.28.18.

X u T.S., Gu L.H., Zhang X.H., Hu ang W., Ye B.G., Li u X.L., Hou S.S. (2013 b). IGF-1 and FoxO3 expression profiles and developmental differences of breast and leg muscle in Pekin ducks during postnatal stages. J. Anim. Vet. Adv., 12: 852-858.

X u T.S., Gu L.H., S un Y., Zhang X.H., Ye B.G., Li u X.L., Hou S.S. (2015). Characterization of MUSTN1 gene and its relationship with skeletal muscle development at postnatal stages in Pekin ducks. Genet. Mol. Res., 14: 4448-4460; https://doi.org/10.4238/2015.May.4.2.

X u T.S., G u L.H.,H u a n g W., X i a W.L., Z h a n g Y.S.,Z h ang Y.G., R on g G., S c h a c h t s c hne i d e r K., H o u S.S. (2017). Gene expression profiling in Pekin duck embryonic breast muscle. PLoS One, 12: 1-18; https://doi.org/10.1371/journal.pone.0174612.

Y a ffe D., S a x e 1 O. (1977). A myogenic cell line with altered serum requirements for differentiation. Differentiation, 7: 159-166; https://doi.org/10.1111/j.1432-0436.1977.tb01507.x.

Yin H., Pasut A., Sole imani V.D., Bentzinger C.F., Antoun G., Thorn S., Seale P., Fernando P., Van Ijcken W., Grosveld F., Dekemp R.A., Boushel R., Harper M.E., Rudnicki M.A. (2013). MicroRNA-133 controls brown adipose determination in skeletal muscle satellite cells by targeting Prdm16. Cell Metab., 17: 210-224; https://doi. org/10.1016/j.cmet.2013.01.004.

Y in H., Zh an g S., Gil b e r t E.R., S i e g e 1 P.B., Z h u Q., Won g E.A. (2014). Expression profiles of muscle genes in postnatal skeletal muscle in lines of chickens divergently selected for high and low body weight. Poultry Sci., 93: 147-154; https://doi.org/10.3382/ps.2013-03612.

Zhang J., Ying Z.Z., Tang Z.L., Long L.Q., Li K. (2012). MicroRNA-148a promotes myogenic differentiation by targeting the ROCK1 gene. J. Biol. Chem. 287: 21093-21101; https://doi. org/10.1074/jbc.M111.330381.

Zhao Y., Samal E., S rivastava D. (2005). Serum response factor regulates a muscle-specific microRNA that targets Hand2 during cardiogenesis. Nature, 436: 214-220; https://doi.org/10.1038/ nature03817.

Zh a o Y., Hou Y., Z hang K., Yu a n B., P eng X. (2017). Identification of differentially expressed miRNAs through high-throughput sequencing in the chicken lung in response to Mycoplasma gallisepticum HS. Comp. Biochem. Physiol. - Part D Genomics Proteomics, 22: 146-156; https://doi. org/10.1016/j.cbd.2017.04.004.

Zhu C., S ong W., Ta o Z., Li u H., X u W., Zhang S., Li H. (2017). Deep RNA sequencing of pectoralis muscle transcriptomes during late-term embryonic to neonatal development in indigenous Chinese duck breeds. PLoS One, 12: 1-18; https://doi.org/10.1371/journal.pone.0180403.

Received: 24 IV 2019

Accepted: 25 VII 2019 